\title{
Chondroprotective and Anti-Inflammatory Activities of Extracts from Semen Sojae Germinatum on IL-1 $\beta$ - Stimulated Human Osteoarthritis Chondrocytes
}

\author{
Wenhui Fan1, Ying Qian1, Jun Wang1, Xiliang Yang1, Tong Gui ${ }^{2 \star}$ and Bingshu $\mathrm{He}^{2}$ \\ 1Department of Pharmacy, College of Medicine, Wuhan University of Science and Technology, Wuhan-430065, CHINA. \\ ${ }^{2}$ Department of Orthopedic Surgery, Hubei Woman and Child Hospital, Wuhan-430070, CHINA.
}

\begin{abstract}
Our previous study showed that Semen sojae germinatum (SSG), a soy-derived Chinese medicinal material, have potential benefits for knee osteoarthritis (OA). This study was undertaken to identify the major effective sub-fraction of SSG. The $95 \%$ ethanol extract of SSG was successively fractionated into petroleum ether, ethyl acetate, n-butanol and aqueous fractions. Then we examined the effect of fractions on the inflammatory response and cell proliferation of primary human osteoarthritic chondrocytes stimulated by interleukin (IL)-1 $1 \beta$, a recognized and common inducer of OA in vitro. Results showed that the petroleum ether, ethyl acetate and n-butanol extracts of SSG promoted cell-proliferation measured by MTT [3-(4,5- dimethylthiazolyl-2)-2,5-diphenyltetrazoliumbromide] assay, increased the transcript levels and nuclear translocation of cyclin D1, and inhibited the production of pro inflammatory mediator prostaglandin $(P G) E_{2}$ and nitric oxide (NO) induced by IL-1 $\beta$ in human OA chondrocytes, suggesting SSG extracts possessed chondro protective and anti-inflammatory properties. The ethyl acetate fraction was superior to other fractions. These data indicated SSG extracts might become a potential treatment option for humans with OA.
\end{abstract}

Key words: Semen sojae germinatum, Extract, Osteoarthritis, Chondrocytes, Inflammatory response, Proliferation.

\section{INTRODUCTION}

Semen sojae germinatum (SSG) is a traditional Chinese medicine, which is essentially dry and processed bean sprout coming from germinating mature seed of Chinese black soybean (Glycine max L. Merr.) variety. This soy derived medicinal material was recorded by "Shen Nong's Herbal Classic"(earliest pharmacopeia in ancient China which was written as early as 2000 years ago), later "Compendium of Materia Medica" written by Li Shizhen in the Ming Dynasty (CE 1368-1644), and has also been included in the current edition of Pharmacopoeia of People's Republic of China. In "Compendium of Materia Medica", SSG have been recorded as a valuable Chinese single herb for removing "Bi syndrome of bone" and dispelling "wind-hot-dampness", which are important indices for "knee pain" in traditional Chinese medicine.

Knee pain is a common disease affecting about a quarter of people aged over 55 years old, and normal daily activities in about half of patients would be restricted when the pain is severe enough. ${ }^{1}$ After excluding specific conditions such as inflammatory arthritis, an over whelming majority of knee pain is given the label of 'osteoarthritis' (OA). Knee OA is one of the most common degenerative diseases characterized by synovial inflammation and progressive loss of joint cartilage, which
Submission Date : 17-01-2016 Revision Date : :04-04-2016 Accepted Date : :06-04-2016

DOI: 10.5530/ijper.50.3.12 Correspondence: Dr. Tong Gui,

Department of Orthopedic Surgery, HubeiWoman and Child Hospital, Wuhan-430070, CHINA. E-mail:wangj79@hotmail. com

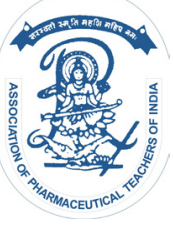

www.ijper.org 
affects millions of aging people worldwide. However, the understanding of $\mathrm{OA}$ as a "wear and tear" disease has been recently shift to an inflammatory disease. ${ }^{2,3}$ Signs and symptoms of localized inflammation such as stiffness and joint effusion are presented as common clinical manifestations of knee OA. On the other hand, the evidence of cell death in osteoarthritic chondrocytes indicates that chondrocyte death/survival also plays a key role in OA pathogenesis. ${ }^{4,5}$ The proliferation and synthetic activity of cartilage and bone cells are generally considered to be involved in the regulation of physiological remodeling and cartilage healing, ${ }^{6}$ mediate repair and protective processes in osteoarthritic cartilage and subchondral cancellous bone, thereby are advantageous to the treatment of OA. ${ }^{7}$ Chondrocytes are the resident cells of articular cartilage, and responsible for the maintenance of extra cellular matrix. A definite negative co-relation has been clinically reported between the number of chondrocytes and the degree of cartilage damage. ${ }^{8}$ Maintaining and promoting chondrocyte proliferation and viability could potentially delay the progression of cartilage degeneration during $\mathrm{OA}$.

The current treatments for $\mathrm{OA}$ (e.g. non steroidal antiinflammatory drugs, cortico steroids or intra-articular hyaluronic acid) are marginal effective even ineffective, or associated with serious adverse events. Under this circumstance, complementary and alternative medicine represented by Chinese herbal medicine has become a popular treatment option for knee OA patients around the world. ${ }^{9,10}$ In our previous study, ${ }^{11}$ to investigate the preliminary effect of SSG on experimental OA, we have demonstrated that prepared SSG as feed could improve cartilage degeneration and cartilage matrix degradation, decrease the levels of inflammatory mediators in synovial fluid of osteoarthritic rabbit knee joints, suggesting SSG showed potential benefits for knee OA. Nevertheless, the active components of SSG and their pharmacological activities are still unclear. In the present study, we examined the effect of fractions extracted from SSG on the inflammatory response and cell proliferation of primary human osteoarthritic chondrocytes stimulated by inter leukin(IL)-1 $\beta$, a recognized and common inducer of $\mathrm{OA}$ in vitro. ${ }^{2}$

\section{MATERIALS AND METHODS}

\section{Preparation of the extracts from SSG}

As described in our previous study, ${ }^{11}$ SSG was prepared from sprouted Chinese black soybeans (Glycine max L. Merr.) referring to Pharmacopoeia of People's Republic of China (Edition 2010). Dried prepared SSG (1500 g) were crashed then extracted by reflux extraction with
$95 \%$ ethanol at $50^{\circ} \mathrm{C}$ for $2-3 \mathrm{~h}$. And this extraction step was repeated three times. The extracted liquid was pooled, filtered then concentrated at $50^{\circ} \mathrm{C}$ using a rotary evaporator. The decoction was vacuum dried to obtain the concentrated residue, which was the crude extract from SSG (46.5g).

Then, the crude extract were suspended in distilled water and partitioned sequentially with petroleum ether, ethyl acetate, and n-butanol to obtain respective fractions. Each organic fraction was then evaporated to dryness separately, and yielded a petroleum ether extract (PEE) (4.0 g), an ethyl acetate extract (EAE) $(3.2 \mathrm{~g})$, and an n-butanol extract (NBE) (6.1 g), while the remaining was aqueous fractions (AF) of SSG $(14.0 \mathrm{~g})$. Each extract were respectively dissolved in dimethyl sulfoxide at a concentration of $10,000 \mu \mathrm{g} / \mathrm{ml}$ as stock solution.

\section{Chondrocyte culture and treatment}

Human osteoarthritic chondrocytes were isolated from pooled cartilage samples obtained from the femoral chondyle and tibia plateau of 4 female OA patients undergoing total knee arthroplasty. The average age of patients was 68.7 years (range of 65-71). OA was diagnosed according to the Diagnostic and Therapeutic Criteria Committee of the American Rheumatism Association. ${ }^{13}$ This study was approved by the Ethics Committee of Hubei Woman and Child Hospital, and informed consent was obtained from each patient. Cartilage was incubated with $1 \mathrm{mg} / \mathrm{ml}$ trypsin (Sigma, MI, USA) for 1 hour followed by an overnight digestion in $0.5 \mathrm{mg} / \mathrm{ml}$ collagenase type II (Worthington, Lakewood, $\mathrm{NJ}$ ) in a $37^{\circ} \mathrm{C}$ water bath. The resulting cell suspension was filtered twice using $70 \mu \mathrm{m}$ nylon meshes, washed, and then centrifuged for $10 \mathrm{~min}$ at $700 \times \mathrm{g}$. Trypan blue viability test showed $90 \%-95 \%$ of the recovered cells were alive. Primary cells cultures were seeded at $1-2 \times 10^{5}$ cells $/ \mathrm{ml}$ in a $25 \mathrm{~cm}^{2}$ flask at $37^{\circ} \mathrm{C}, 5 \% \mathrm{CO}_{2}$ in DMEM/ F12 medium supplemented with 10\% fetal calf serum (FCS), $100 \mathrm{U} / \mathrm{mL}$ penicillin and $100 \mathrm{U} / \mathrm{ml}$ streptomycin. All chondrocytes were used for the experiments at the second passage. OA chondrocytes were then seeded into six-well plates at $6 \times 10^{5}$ cells $/$ well and incubated for 24 hours. Culture medium was then replaced with serum starved media ( $0.5 \%$ FCS) and exposed to $5 \mathrm{ng} / \mathrm{ml} \mathrm{IL}-1 \beta$ (Sigma, MI, USA) for $24 \mathrm{~h}$. Untreated cells were used as controls.

\section{Chondrocyte proliferation and viability assay}

The effect of various amounts of different SSG extracts $(1000,500,250,125,62.5,31.3,15.6,7.8$, and $3.9 \mu \mathrm{g} / \mathrm{mL})$ on the chondrocyte proliferation and viability was studied using MT'T (3-(4,5- dimethylthiazolyl-2)-2,5- 
diphe-nyltetrazoliumbromide) assay in accordance with the manufacturer's instructions ( $\mathrm{R} \& \mathrm{D}$ Systems). The resulting absorbance was measured at $570 \mathrm{~nm}$.

Enzyme-Linked Immunosorbent Assay (ELISA) for prostaglandin $(P G) E_{2}$ in culture supernatant produced by chondrocytes

The levels of $\mathrm{PGE}_{2}$ released from cultured chondrocytes were detected by specific ELISA kit (R\&D Systems, Inc., Minneapolis, MN, USA) according to the instructions of the manufacturer.

\section{Measurement of nitric oxide (NO) in culture supernatant produced by chondrocytes}

The concentration of $\mathrm{NO}$ in culture supernatant produced by chondrocytes was measured by Griess reagent according to the instructions of the manufacturer (Jiancheng, Nanjing, China). Detection of NO concentration was performed by estimating the level of nitrite, a NO metabolite.

\section{Quantitative real-time polymerse chain reaction (PCR)}

Total RNA of monolayer cultured osteoarthritic chondrocytes was isolated using TRIzol Reagent (In vitro gen, USA). After quantifying the isolated RNA using a spectrophotometer, 2-lg aliquots were reverse transcribed using a Transcript or First Strand c DNA Synthesis Kit (Genecopoiea, USA.). The primer sequences of Cyclin D1 are 5'-TCTACACCGACAACTCCATCCG-3' (forward) and 5'-TCTGG CATTTTGGAGAGGAAGTG-3' (reverse). Real-time PCR was carried out in an Applied Biosystems Step One Plus ${ }^{\mathrm{TM}}$ Real-Time PCR System. Levels of cyclin D1 mRNA were normalized to those of $\beta$-actin.

\section{Immuno fluorescence}

Cells were stained with the anti-cyclin D1 primary antibodies, followed by peroxidase-conjugated secondary antibodies (Southern Biotech, Birmingham, Al). Immunostains were visualized using a fluorescent tyramide reagent (TSA-direct NEL-701, PerkinElmer, Waltham, $\mathrm{MA})$.

\section{Statistical analysis}

The statistical software package SPSS 12.0 was used. The data are presented as the means \pm S.D. of five independent experiments. The statistical significance of differences was determined by one-way analysis of variance. $\mathrm{p}<0.05$ was considered to indicate statistical significance.

\section{RESULTS}

\section{Effect of SSG extracts on chondrocyte proliferation and viability}

To explore the effect of SSG extracts on chondrocyte growth and determine their appropriate concentration ranges, MTT assay was performed on chondrocytes for the detection of cell proliferation and viability following exposure to different extracts. As shown in Figure 1, PEE at the concentrations of $62.5,125$ and $250 \mu \mathrm{g} / \mathrm{mL}$ showed $23.7 \%, 28.9 \%$ and $31.6 \%$ increase of chondrocyte proliferation and viability, respectively $(p<0.05)$. The concentration of EAE at $62.5-250 \mu \mathrm{g} / \mathrm{m}$ and NBE at $250 \mu \mathrm{g} / \mathrm{mL}$ respectively showed significant protection for chondrocytes against IL-1 $\beta$-induced damage $(p<0.05$; $p<0.01)$. These results indicated that PEE, EAE and NBE might be responsible for the growth promoting effect of SSG on OA chondrocytes. Despite the fact that no significant effect of $\mathrm{AF}$ was found, the concentration range from 62.5 to $250 \mu \mathrm{g} / \mathrm{mL}$ was appropriate for most of the extracts, especially, a concentration of $125 \mu \mathrm{g} / \mathrm{ml}$ was acceptable in most cases.

To confirm the effect of SSG extracts on chondrocyte proliferation, the mRNA expression level of cyclin D1, a major cell cycle regulatory switch in proliferating cells, in OA chondrocytes treated with SSG extracts at concentration of $62.5-250 \mu \mathrm{g} / \mathrm{mL}$ was measured with Real-time PCR, and subcellular locations of cyclin D1 in chondrocytes treated with $125 \mu \mathrm{g} / \mathrm{mL}$ different extracts were determined by immunofluorescence staining. As shown in Figure 2A, PEE and NBE at concentration of 250 $\mu \mathrm{g} / \mathrm{mL}$ increased the mRNA expression level of cyclin D1 in IL-1 $\beta$-induced human osteoarthritic chondrocytes $(p<0.05)$. Among the different solvent fractions, EAE was remarkably effective one in upregulating cyclin D1 expression of OA chondrocytes, which was manifested as $50.0-92.7 \%$ increase at concentrations of $62.5-250 \mu \mathrm{g} / \mathrm{mL}(p<0.05 ; p<0.01)$. Cyclin D1 immuno reactivity was found mainly in the cytoplasm in control and IL-1 $\beta$-induced chondrocytes. And further increased nuclear cyclin D1 were detected in cells treated with $125 \mu \mathrm{g} / \mathrm{mL}$ EAE (Figure 2B).

\section{Effect of SSG extracts on IL-1 $\beta$-induced PGE and NO production in human osteoarthritic chondrocytes}

$\mathrm{PGE}_{2}$, as an important pro inflammatory mediator, has long been demonstrated to be one of the major catabolic mediators involved in cartilage degradation that push forward the progression of $\mathrm{OA}$ disease. ${ }^{14} \mathrm{The}_{\mathrm{PGE}}$ level in the supernatant was assessed using an ELISA assay. As shown in Figure 3, upon IL-1 $\beta$ stimulation 

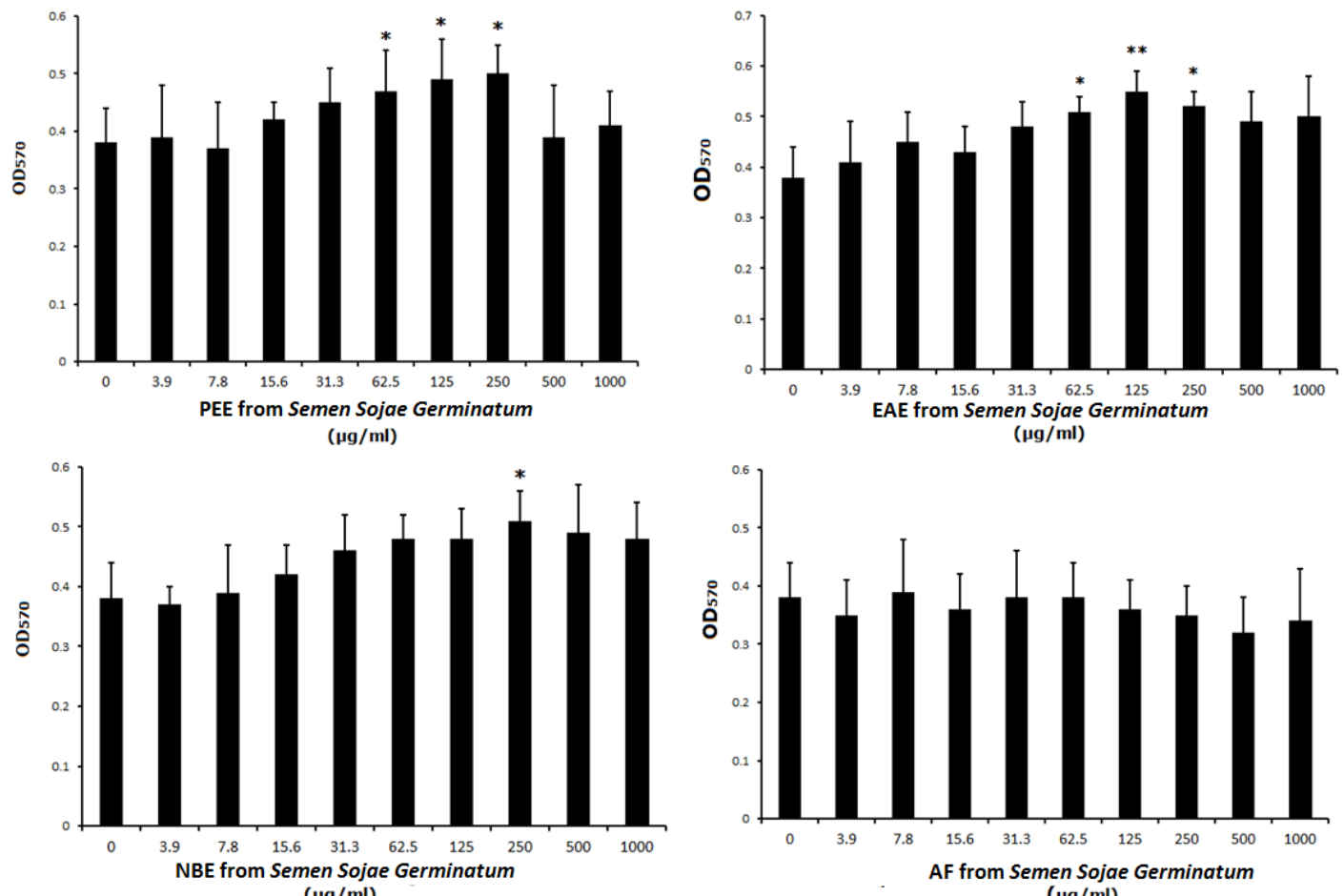

$(\mu \mathrm{g} / \mathrm{ml})$

Figure 1: Effects of SSG extracts on chondrocyte proliferation and viability of IL-1 $\beta$-stimulated human osteoarthritic chondrocytes. The proliferation and viability of chondrocytes was studied using the MTT assay. ${ }^{*} \mathrm{p}<0.05,{ }^{* *} \mathrm{p}<0.01$, compared with IL-1 $\beta$-stimulated human osteoarthritic chondrocytes. $n=5$ in each group.

A

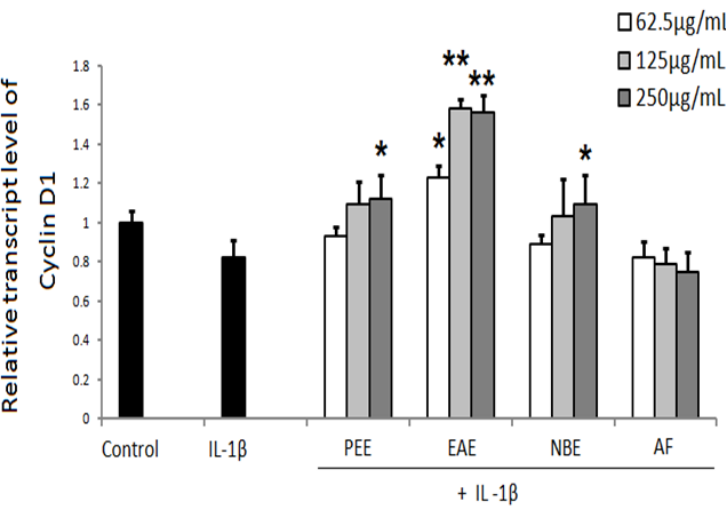

B

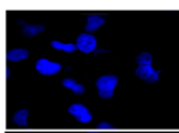

Cyclin D1

Merged

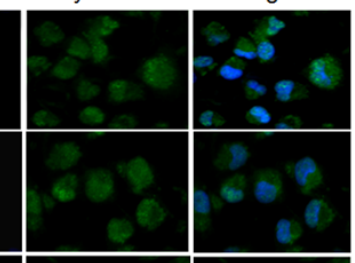

PEE+

IL-1 $\beta$
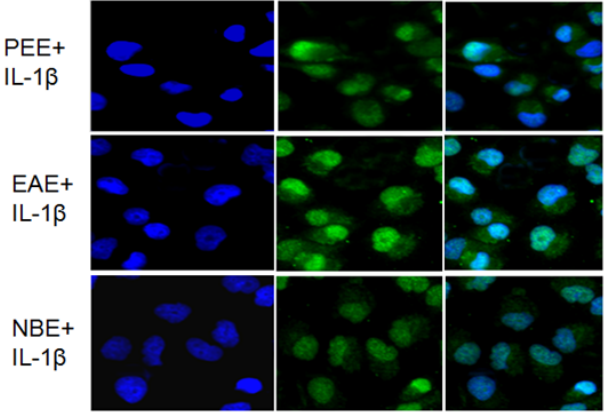

$\mathrm{AF}+$

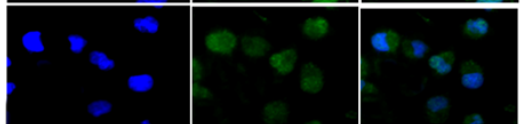

Figure 2: Effects of SSG extracts on Cyclin D1, a major cell cycle regulatory switch in proliferating cells, in IL-1 $\beta$-stimulated human osteoarthritic chondrocytes. A: Transcript levels of cyclin D 1 was determined by real-time PCR. B: The subcellular locations of cyclin D1 were determined by immunofluorescence staining.

${ }^{*} p<0.05,{ }^{* *} p<0.01$, compared with IL-1 $\beta$-stimulated human osteoarthritic chondrocytes. $n=5$ in each group. PEE: petroleum ether extract; EAE: ethyl acetate extract; NBE: n-butanol extract; AF:aqueous fractions. 
$\square 62.5 \mu \mathrm{g} / \mathrm{mL}$ $\square 125 \mu \mathrm{g} / \mathrm{mL}$

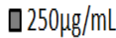
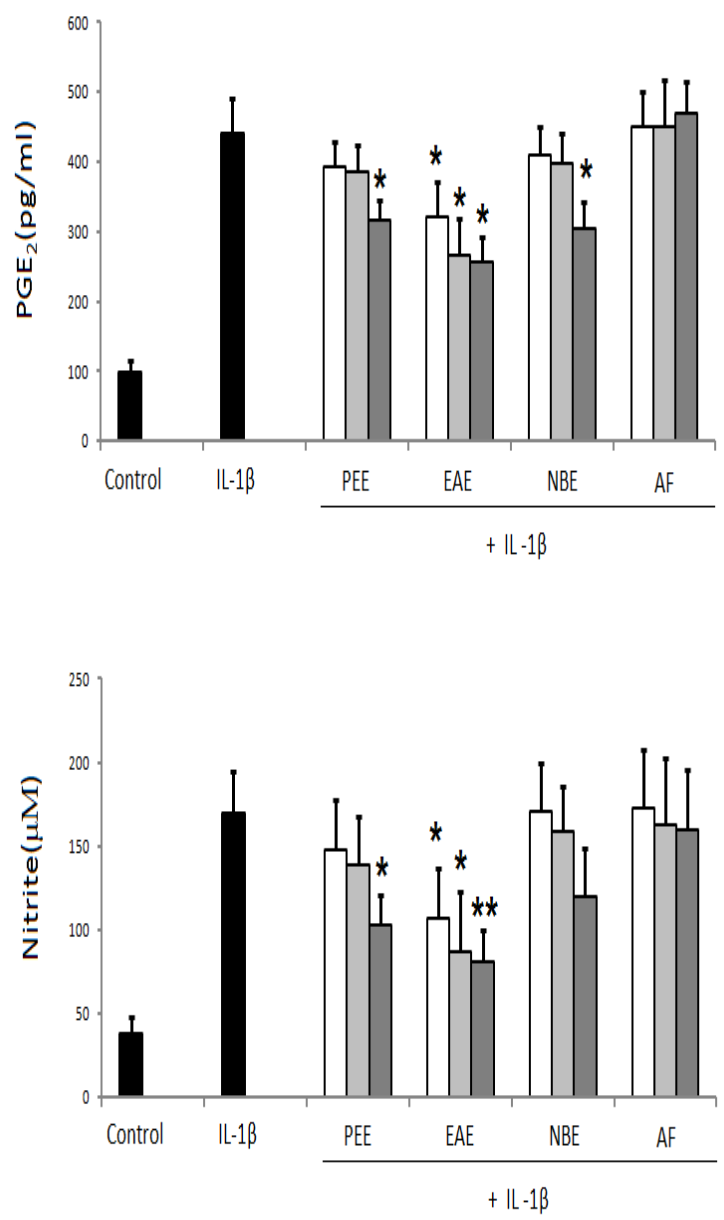

Figure 3: Effects of SSG extracts on $\mathrm{PGE}_{2}$ and NO production of IL-1 $\beta$-stimulated human osteoarthritic chondrocytes. PGE and NO levels in the supernatant were assessed using ELISA assay and Griess method, respectively.

${ }^{*} p<0.05,{ }^{* *} p<0.01$, compared with IL-1 $\beta$-stimulated human osteoarthritic chondrocytes. $n=5$ in each group. PEE: petroleum ether extract; EAE: ethy acetate extract; NBE: n-butanol extract; AF:aqueous fractions.

( $5 \mathrm{ng} / \mathrm{ml}$ ), chondrocytes produced over 5 times more $\mathrm{PGE}_{2}$ than unstimulated chondrocytes $(p<0.01)$. Importantly, treatment of chondrocytes with PEE, EAE and $\mathrm{NBE}$ further reduced the $\mathrm{PGE}_{2}$ release induced by IL-1 $\beta$ stimulation $(p<0.05 ; p<0.01)$. Over production of NO have also been recognized as an inflammatory mediator involved in the pathophysiology of OA. Increased NO levels in plasma have been reported in patients with OA. ${ }^{15,16}$ More over, serum NO content was found correlated with pain scores in response to pressure, total Lequesne's scores, and swelling scores of OA, thus has been believed as a biochemical factor implicated in joint degeneration. ${ }^{16}$ We found PEE and EAE of SSG were effective in attenuating IL- $1 \beta$-induced NO overproduction in chondrocytes $(p<0.05 ; p<0.01$, Figure 3). In general, these results demonstrated that PEE, EAE and NBE from SGG might serve to control and resolve inflammation and cartilage degradation during $\mathrm{OA}$, and $\mathrm{EAE}$ was more active than the other extracts.

\section{DISCUSSION}

Accumulating evidences have suggested that soy derived products were safe and beneficial in the management of OA. ${ }^{17-19}$ The efficacy of soy protein supplementation in relieving the pain and discomfort associated with $\mathrm{OA}$ has been reported in a parallel, randomized, doubleblind, placebo-controlled study. ${ }^{17}$ Specific components of soy, such as genistein, daidzein and glycitein, have been shown exert protective effects via reducing inflammation or modulating cartilage metabolism in various OA models in vitro or in vivo. ${ }^{18}$ Although there was scant evidence about the effect of soy isoflavone in the setting of $\mathrm{OA}$, the anti-inflammatory properties of isoflavones have been well-described, ${ }^{20}$ and also provide the rationale for the protective effect of soy derived products in conditions such as OA. ${ }^{17}$ In addition, soybean unsaponifiables as other components of soy have been shown to modulate $\mathrm{OA}$ pathogenesis by inhibiting catabolic pathways, fibrinolysis and inflammatory cytokines, promoting cartilage repair, and could reduce stiffness and pain while improving joint function at the clinical level. ${ }^{19}$ Since SSG essentially is a kind of processed bean sprout, these above researches further support the potential to use SSG and its extracts as protective agents against OA. As a rheumatism-dispelling agent, SSG has been used for the control of knee joint pain and arthritis in Chinese medicine for over 2000 years. Based on our previous study on the protective effect of SSG against the development of knee OA, ${ }^{11}$ this study has conducted a pharmacological activity screen on the extracts got from SSG looking at the chondro protective and anti-inflammatory activities in an in vitro model of $\mathrm{OA}$. We further confirmed that, overall, the petroleum ether, ethyl acetate and n-butanol extracts of SSG promoted cell-proliferation measured by MTT assay, increased the transcript levels and nuclear translocation of cyclin D1, and inhibited the production of pro inflammatory mediator $\mathrm{PGE}_{2}$ and $\mathrm{NO}$ induced by $\mathrm{IL}-1 \beta$ in human $\mathrm{OA}$ chondrocytes, suggesting extracts from SSG possessed chondro protective and anti-inflammatory properties. These data indicated that, being soy-derived safe products, the extracts of SSG might become a potential treatment option for humans with OA. There into, the ethyl acetate fraction was superior to other fractions in protecting chondrocytes and reducing IL-1 $\beta$-induced inflammation. However, the aqueous fractions had almost no chondrocyte protection and 
anti-inflammatory effect. The different activities of diverse polar solvents extracts of SSG might be due to the differences in compositions. Therefore, further phyto chemical investigations to determine the active molecules in each of the tested extracts are needed to underline the outcome. And it could be deduced that the ethyl acetate fraction contains high amount of the constituents which are responsible for the protective effect against OA. Besides, only one time point (incubation of chondrocytes with extracts for 24 hours) was selected in this study to evaluate the chondro protective and antiinflammatory activities of different extracts from SSG at different doses. Based on the preliminary results from the present study at one time point, further researches were also needed to assess the activity of active extracts such as PEE, EAE and NBE from SGG on a longer/ different time points, in order to reflect the definitive activity of the extracts.

In addition, as a soy derived product and widely-used Chinese medicine, SSG ought to possess an excellent safety profile. Accordingly, in the present study, MTT assay also showed all the extracts of SSG cause no direct cytotoxicity on human chondrocytes.

\section{CONCLUSION}

Results from this study showed that the petroleum ether, ethyl acetate and n-butanol extracts of SSG promoted cell-proliferation, increased the transcript levels and nuclear translocation of cyclin D1, and inhibited the production of pro inflammatory mediator $\mathrm{PGE}_{2}$ and NO induced by IL-1 $\beta$ in human OA chondrocytes, suggesting SSG extracts possessed chondro protective and anti-inflammatory properties. These data may provide valuable evidence for the traditional use of SSG in removing "Bi syndrome of bone" and dispelling "windhot-dampness", indicate SSG extracts might become a potential treatment option for humans with OA. The ethyl acetate fraction was superior to other fractions. It would be of great interest to further elucidate the structures of components responsible for the protective effect against $O A$.

\section{ACKNOWLEDGEMENT}

This research has been financially supported by Provincial College Students' Innovative Entrepreneurial Training Program in Hubei (201310488044).

\section{CONFLICTS OF INTEREST}

There is no conflict of interest for the present communication.

\section{REFERENCES}

1. White A, Foster NE, Cummings $M$ and Barlas P. Acupuncture treatment for chronic knee pain: a systematic review. Rheumatology. 2007;46(3):384-90.

2. Daghestani HN and Kraus VB. Inflammatory biomarkers in osteoarthritis. Osteoarthritis Cartilage. 2015;23:1890-6.

3. Wojdasiewicz P, Poniatowski $Ł A$ and Szukiewicz D. The role of inflammatory and anti-inflammatory cytokines in the pathogenesis of osteoarthritis. Mediators Inflamm. 2014;2014:561459.

4. Musumeci G, Castrogiovanni P, Trovato FM, Weinberg AM, Al-Wasiyah MK, Alqahtani $\mathrm{MH}$ and Mobasheri A. Biomarkers of Chondrocyte Apoptosis and Autophagy in Osteoarthritis. Int J Mol Sci. 2015;16(9):20560-75.

5. Hwang HS and Kim HA. Chondrocyte Apoptosis in the Pathogenesis of Osteoarthritis. Int J Mol Sci. 2015;16:26035-54.

6. Civinini R, Nistri L, Martini C, Redl B, Ristori G and Innocenti M. Growth factors in the treatment of early osteoarthritis. Clin Cases Miner Bone Metab. 2013;10(1):26-9.

7. Mentlein R. Targeting pleiotropin to treat osteoarthritis. Expert Opin Ther Targets. 2007;11(7):861-7.

8. Loeser RF, Pacione CA and Chubinskaya S. The combination of insulin-like growth factor 1 and osteogenic protein 1 promotes increased survival of and matrix synthesis by normal and osteoarthritic human articular chondrocytes. Arthritis Rheum. 2003;48(8):2188-96.

9. Hou PW, Fu PK, Hsu HC and Hsieh CL. Traditional Chinese medicine in patients with osteoarthritis of the knee. J Tradit Complement Med. 2015;5:182-96.

10. Zhu L, Yang S, Wang S, Gong H, Li L and Wei X. Effectiveness and Safety of Manufactured Chinese Herbal Formula for Knee Osteoarthritis: Insights from a Systematic Review. Evid Based Complement Alternat Med. 2015;2015:328642.

11. He B, Wang J. Effect of Semen sojae germinatum on experimental osteoarthritis in the rabbit knee. Bangladesh J Pharmacol. 2013;8(4):365-70.

12. Liu L, Gu H, Liu H, Jiao Y, Li K, et al. Protective effect of resveratrol against IL-1 $\beta$-induced inflammatory response on human osteoarthritic chondrocytes partly via the TLR4/MyD88/NF-KB signaling pathway: an "in vitro study". Int J Mol Sci. 2014;15(4):6925-40.

13. Altman R, Asch E, Bloch D, Bole G, Borenstein D, et al. Development of criteria for the classification and reporting of osteoarthritis. Classification of osteoarthritis of the knee. Diagnostic and Therapeutic Criteria Committee of the American Rheumatism Association Arthritis Rheum. 1986;29(8):1039-49.

14. Martel-Pelletier J, Pelletier JP and Fahmi H. Cyclooxygenase-2 and prostaglandins in articular tissues. Semin Arthritis Rheum. 2003;33(3):15567.

15. Salvatierra J, Escames G, Hernandez P, Cantero J, Crespo E, Leon J, Salvatierra D, Acuña-Castroviejo D and Vives F. Cartilage and serum levels of nitric oxide in patients with hip osteoarthritis. J Rheumatol. 1999;26(9):2015-7.

16. Li H, Li L, Min J, Yang $H, X u X$, Yuan $Y$ and Wang D. Levels of metalloproteinase (MMP-3, MMP-9), NF-kappaB ligand (RANKL), and nitric oxide (NO) in peripheral blood of osteoarthritis (OA) patients. Clin Lab. 2012;58(7-8):755-62.

17. Arjmandi BH, Khalil DA, Lucas EA, Smith BJ, Sinichi N, Hodges SB and Svanborg A. Soy protein may alleviate osteoarthritis symptoms. Phytomedicine. 2004;11(7):567-75.

18. Shen CL, Smith BJ, Lo DF, Chyu MC, Dunn DM, Chen $\mathrm{CH}$ and Kwun IS. Dietary polyphenols and mechanisms of osteoarthritis. J Nutr Biochem. 2012;23(11):1367-77.

19. Christiansen BA, Bhatti S, Goudarzi R and Emami S. Management of Osteoarthritis with Avocado/Soybean Unsaponifiables. Cartilage. 2015;6(1):30-44.

20. Dijsselbloem N, Vanden Berghe W, De Naeyer A, Haegeman G. Soy isoflavone phyto-pharmaceuticals in interleukin- 6 affections. Multi-purpose nutraceuticals at the crossroad of hormone replacement, anti-cancer and anti-inflammatory therapy. Biochem Pharmacol. 2004;68(6):1171-85. 